\title{
Fully-compliant statically-balanced mechanisms without prestressing assembly: concepts and case studies
}

\author{
G. Chen and S. Zhang
}

School of Mechatronics, Xidian University, Xi' an, Shaanxi 710071, China

Received: 22 January 2011 - Revised: 1 August 2011 - Accepted: 5 August 2011 - Published: 16 August 2011

\begin{abstract}
The purpose of this paper is to present new concepts for designing fully-compliant staticallybalanced mechanisms without prestressing assembly. A statically-balanced compliant mechanism can ideally provide zero stiffness and energy free motion like a traditional rigid-body mechanism. These characteristics are important in design of compliant mechanisms where low actuation force, accurate force transmission or high-fidelity force feedback are primary concerns. Typically, static balancing of compliant mechanisms has been achieved by means of prestressing assembly. However, this can often lead to creep and stress relaxation arising in the flexible members. In this paper two concepts are presented which eliminate the need for prestressing assembly of compliant mechanisms: (1) a weight compensator which employs a constant-force compliant mechanism, (2) a near-zero-stiffness mechanism which combines two multistable mechanisms. In addition to the advantages provided by statically-balanced compliant mechanisms, two other notable features of these statically-balanced mechanisms are their ability to be monolithically fabricated and to return to their as-fabricated position without any disassembly when not in use.
\end{abstract}

\section{Introduction}

Compliant mechanisms, which utilize the deflection of flexible segments rather than from articulated joints to achieve their mobility, offer many advantages over their rigid-body counterparts such as decreased part count, increased precision and reduced wear (Howell, 2001). Because a compliant mechanism obtains its motion from deflection of its members, spring-back forces play a significant role in its input and output capabilities. Hence the study of the force-deflection characteristics (Jutte and Kota, 2008) of a compliant mechanism is among the most important methods of understanding its behavior.

Compliant mechanisms can be roughly divided into four categories depending on their force-deflection characteristics: spring-like compliant mechanisms (Trease et al., 2005), multistable compliant mechanisms (Oh and Kota, 2009; Gerson et al., 2008; Chen et al., 2011b, 2010), constantforce compliant mechanisms (Nahar and Sugar, 2003; Lan et al., 2010), and statically-balanced compliant mechanisms (Herder and van den Berg, 2000; Gallego and Herder, 2010). Statically-balanced compliant mechanisms may be consid-

Correspondence to: G. Chen (guimin.chen@gmail.com) ered a constant-force compliant mechanism with zero force input. The design approaches of statically balanced mechanisms may be used to design constant-force mechanisms (Gallego and Herder, 2010).

The concept of a statically balanced compliant mechanism was first introduced and studied by Herder and van den Berg (2000). A statically balanced compliant mechanism, which utilizes energy-release elements to balance the energy stored in the flexible segments of the mechanism, maintains neutral equilibrium throughout its range of motion (Hoetmer et al., 2009; Gallego and Herder, 2010). Like their traditional rigid body counterparts, these mechanisms provide energy free motion with zero accompanying stiffness - a fact particularly useful in cases where accurate force transmission and high-fidelity force feedback are of primary concern in compliant mechanisms (Hoetmer et al., 2009).

Most of the statically balanced compliant mechanisms rely on prestressing to achieve static balancing (Trease and Dede, 2011; Powell and Frecker, 2005; Hoetmer et al., 2009). However implementation difficulty, creep and stress relaxation of flexible members are the challenges associated with prestressing. Accurate prestressing is difficult to achieve in practice, particularly in micromechanical devices due to the small available operating space (Tolou et al., 2010). Moreover, creep and stress relaxation arising from prestressing may dramatically deteriorate the performance of the mechanism 
(Howell, 2001). In contrast, less work has been done on statically-balanced mechanisms without prestressing assembly, although they receive increasing attention. For example, Stapel and Herder (2004) presented a feasibility study of a fully compliant statically balanced grasper in 2004, and Tolou et al. (2010) presented two concepts of fully-compliant statically-balanced compliant micro mechanisms and validated them through simulation in 2010.

In this paper two novel concepts of fully-compliant statically balanced compliant mechanisms are proposed which eliminate the need for prestressing assembly altogether. The first concept is a weight compensator using a constant-force compliant mechanism, and the second is a near-zero-stiffness mechanism based on combination of two multistable mechanisms. Two design cases are presented to demonstrate the feasibility of the concepts. In addition to the advantages such as energy efficiency, accurate force transmission and actuator effort reduction provided by statically balanced compliant mechanisms (Radaelli et al., 2010), they exhibit two other key features: they are able to be monolithically fabricated, and they return to their as-fabricated position without any disassembly when not in operation.

The rest of this paper is organized as follows: Section 2 presents a gravity compensator based on a fully compliant constant-force mechanism. Section 3 studies how to achieve static balancing by combining two multistable mechanisms. Section 4 provides concluding remarks.

\section{Gravity compensation using constant-force mechanisms}

In this section, we take humanoid robot as an example to illustrate gravity compensation.

Because the weight of a humanoid robot can seriously degrade its dynamic performance and result in energyinefficient operation (Wongratanaphisan and Cole, 2008), gravity compensation is often employed to eliminate or minimize the effects of gravity. The resulting gravity compensated robot is a typical example of a statically balanced mechanism. Because the effect of gravity on the body is constant in the vertical direction, we propose a concept of using a constant-force compliant mechanism to support and compensate for the weight, as illustrated in Fig. 1. This is possible because a constant-force compliant mechanism is a mechanism that produces a constant output force over a range of motion (Nahar and Sugar, 2003).

\subsection{Design of constant-force mechanism for gravity compensation}

It is observed that the fully compliant end-effector shown in Fig. 1a (Chen et al., 2009b) exhibits a constant-force behavior. Based on the pseudo-rigid-body model (PRBM) shown

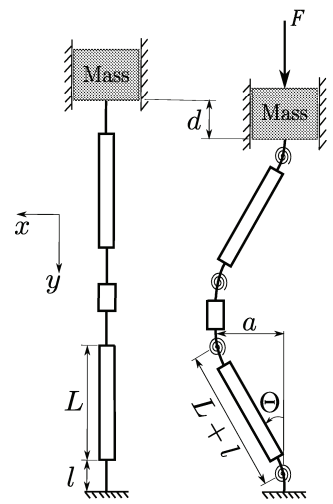

(a)

(b)

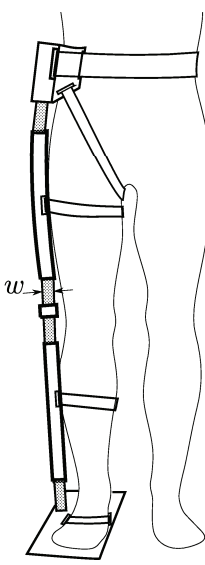

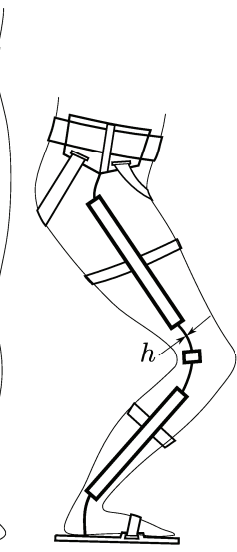

(c)
Figure 1. (a) Schematic diagram of a constant-force mechanism and a mass to be balanced, (b) the corresponding pseudo-rigid-body model (PRBM), and (c) an implementation example for humanoid robot.

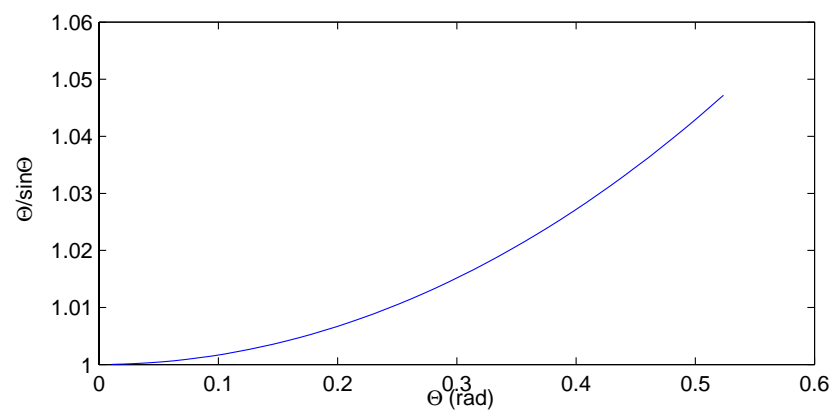

Figure 2. $\Theta / \sin \Theta$.

in Fig. 1b, its force-deflection relationship is given as (Chen et al., 2009b)

$F=\frac{2 K \Theta}{(L+l) \sin \Theta}=\frac{2 K}{L+l} \cdot \frac{\Theta}{\sin \Theta}=\frac{4 K \arccos \left[1-\frac{d}{2(L+l)}\right]}{\sqrt{4(L+l) d-d^{2}}}$

where, $K=E I / l, \Theta$ is the pseudo-rigid-body angle, $L+l$ the length of the pseudo-rigid-body link, $E$ the Young's modulus of the material, and $I$ the moment of inertia of the smalllength flexural pivots. Note that the term $[2 K /(L+l)]$ in Eq. (1) is constant for a specific design, and the change of $\left(\Theta / \sin \Theta\right.$ ) is very small over the range of $0<\Theta \leq 30^{\circ}$ (less than $5 \%$ ), as illustrated in Fig. 2. This indicates that the mechanism acts as a near constant-force, gravity balancing mechanism, and Eq. (1) can be simplified as

$F \approx \frac{2 K}{L+l}$

In this paper a gravity compensator is referred to as a passive mechanical device that counteracts the gravity of a humanoid robot body to improve its dynamic performance 


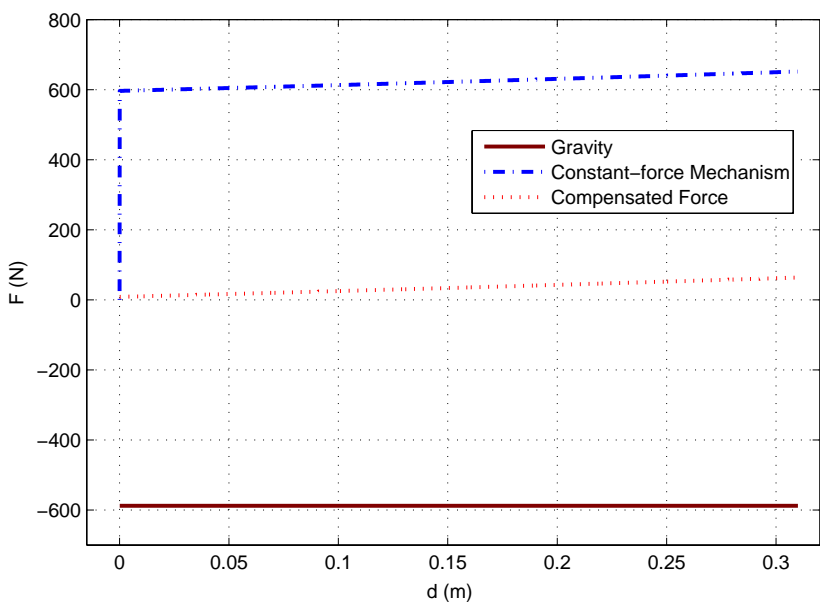

Figure 3. The force-displacement characteristics of the gravity compensated robot. Note that the force-displacement curve for the constant-force mechanism is plotted using Eq. (1) instead of its simplified version, Eq. (2).

and energy efficiency. The gravity compensator based on the constant-force mechanism is designed to satisfy the following requirement:

$G=N_{\mathrm{p}} F=\frac{2 N_{\mathrm{p}} K}{L+l}$

where $N_{\mathrm{p}}$ is the number of flexible segment sets in parallel.

\subsection{Case study}

This subsection presents an example in order to demonstrate the design procedure of a gravity compensator for a humanoid robot using a constant-force compliant mechanism, as shown in Fig. 1c. We first assume the following: $G=60 \times 9.8 \mathrm{~N}, N_{\mathrm{p}}=2, L=0.6 \mathrm{~m}, l=0.02 \mathrm{~m}$ and $E=$ $2.07 \times 10^{11} \mathrm{~Pa}$ (stainless steel). The torsional stiffness of the small-length flexure pivots, $K$, can be calculated from Eq. (3) ( $K=91.14 \mathrm{Nm}$ in this example). Then the moment of inertia for the flexural pivots is determined using $K=E I / l$. As a result, $w=0.02 \mathrm{~m}$ and $h=0.00175 \mathrm{~m}$ can satisfy the design ( $w$ and $t$ are the width and thickness of the cross-section of the small-length pivots respectively). From Fig. 3 we can see that the gravity is well compensated for by the constant force mechanism, with the maximum error less than $10 \%$ for $d$ from 0 to $0.3 \mathrm{~m}$.

\section{Static balancing using multistable compliant mechanisms}

\subsection{Multistable compliant mechanism as negative stiffness building block}

The deflections of the flexible members often produce positive stiffness in a compliant mechanism. Positive stiffness

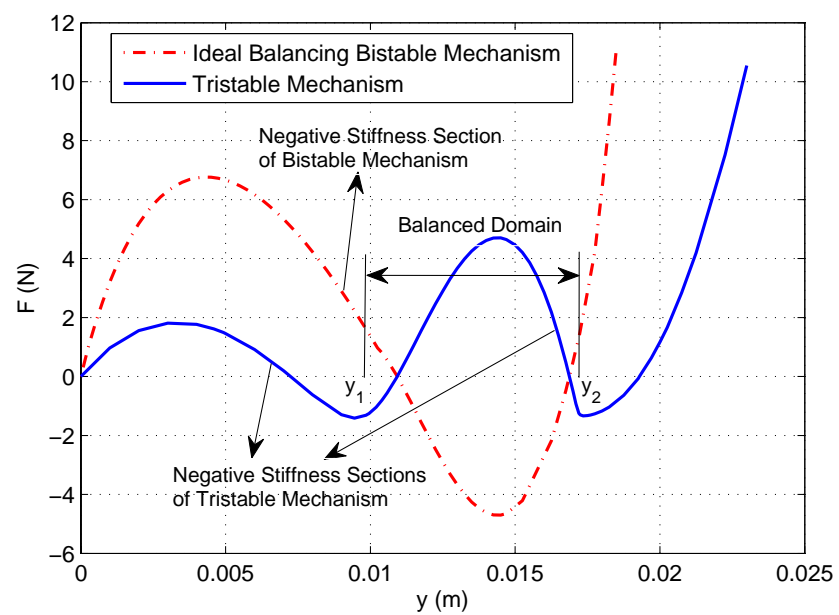

Figure 4. Force-displacement characteristics of an ideal balancing bistable mechanism.

means that the resulting deflection is in the same direction as the external applied force, like the spring-back effect of a linear spring. If a component exhibiting negative stiffness is introduced to the mechanism, the appropriate counterbalancing of positive and negative stiffness in the mechanism can achieve a state of zero-stiffness, i.e., static balancing (Hoetmer et al., 2009).

A bistable mechanism possesses two distinct stable equilibrium positions within its range of motion. At each of these positions the mechanism can maintain stability without power input and, if exposed to a small disturbance while in a stable position, tends to return to the same stable position. It can be seen from Fig. 4 that, for a bistable mechanism, one portion of the force-deflection curve exhibits negative stiffness. Thus Tolou et al. (2010) employed bistable mechanisms as the negative stiffness building blocks in their design of statically-balanced compliant micro mechanisms.

In general, a multistable mechanism with $n$ stable equilibrium positions offers $(n-1)$ negative stiffness sections and $n$ positive stiffness sections on its force-deflection curve (e.g., as shown in Fig. 4, a tristable mechanism possessed two negative stiffness sections and three positive stiffness sections), and as such is a potential building block for synthesis of statically balanced mechanisms. Therefore, we propose a concept for designing fully-compliant staticallybalanced mechanisms which combines two fully compliant multistable mechanisms.

There has been a large amount of work done on multistable mechanisms, including tristable mechanisms (Chen et al., 2009a,b), quadristable mechanisms (Han et al., 2007), and synthesis approaches for multistable mechanisms (Oh and Kota, 2009; Gerson et al., 2008; Chen et al., 2011b). A brief summary of the work on compliant multistable mechanisms can also be found in Chen et al. (2010). Thus this concept may easily be expanded to include these as building blocks 


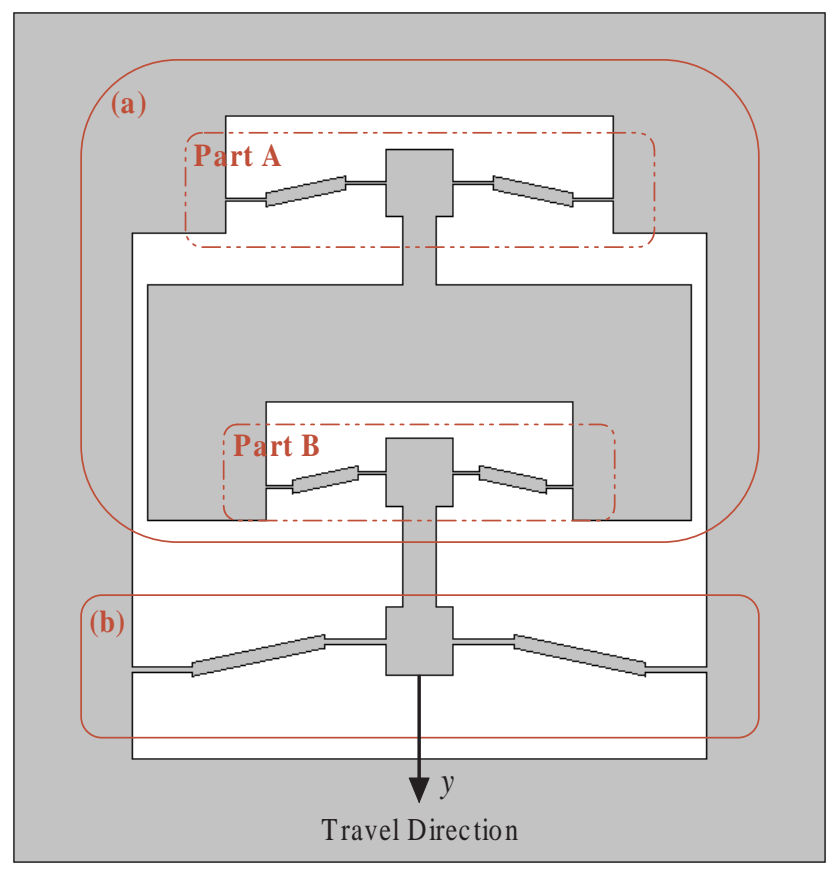

Figure 5. Schematic diagram of a fully-compliant staticallybalanced mechanism consisting of two multistable building blocks, i.e., a tristable mechanism and a bistable mechanism. (a) The tristable mechanism consisting of two different bistable mechanisms (i.e., Part A and Part B) connected in series, and (b) the balancing bistable mechanism.

in the design of more complex statically-balanced mechanisms. In the following subsection a case study is presented to demonstrate the principle of using multistable mechanisms to achieve static balance.

\subsection{Case study}

In this subsection we present a fully-compliant staticallybalanced mechanism combining a tristable mechanism with a bistable mechanism.

To begin our design example, we first chose a specific tristable mechanism and then found a balancing bistable mechanism through an optimization algorithm. The tristable mechanism employed in this example consists of two bistable mechanisms (i.e., Part A and Part B) of different load thresholds connected in series (Oh and Kota, 2009), as shown in Fig. 5a. The design parameters of each bistable mechanism are shown in Fig. 6 and listed in Table 1. Figure 4 gives the force-deflection characteristics of the tristable mechanism (shown as a blue solid line), which is achieved using nonlinear finite element analysis (the detailed finite element modeling approach for this type of bistable mechanism can be found in Cherry et al. (2008)).

We suppose there exists an ideal balancing bistable mechanism which can counterbalance the given tristable mechanism to a maximum balanced domain. Figure 4 plots

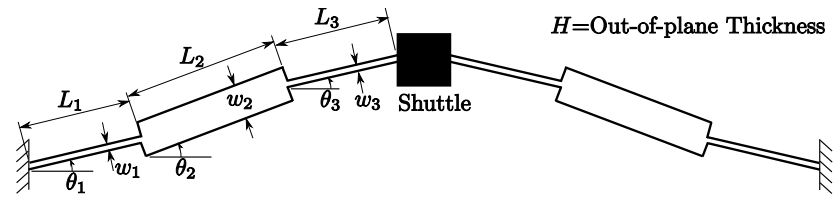

Figure 6. Parameters of bistable mechanism. It should be noted that three bistable mechanisms of this kind are employed in the statically-balanced mechanism shown in Fig. 5, i.e., Part A, Part B and the balancing bistable mechanism.

Table 1. Design values of the tristable mechanism (consisting of two bistable mechanisms connected in series, i.e., Part A and Part B) and the balancing bistable mechanism (see Fig. 6 for the parameters).

\begin{tabular}{crrr}
\hline Parameter & $\begin{array}{r}\text { Part A of } \\
\text { Tristable } \\
\text { Mechanism }\end{array}$ & $\begin{array}{r}\text { Part B of } \\
\text { Tristable } \\
\text { Mechanism }\end{array}$ & $\begin{array}{r}\text { Balancing } \\
\text { Bistable } \\
\text { Mechanism }\end{array}$ \\
\hline$E$ & $1.4 \times 10^{9}$ & $1.4 \times 10^{9}$ & $1.4 \times 10^{9}$ \\
$H$ & $6 \mathrm{~mm}$ & $6 \mathrm{~mm}$ & $6 \mathrm{~mm}$ \\
$L_{1}$ & $12 \mathrm{~mm}$ & $8 \mathrm{~mm}$ & $18 \mathrm{~mm}$ \\
$\theta_{1}$ & $0^{\circ}$ & $0^{\circ}$ & $0^{\circ}$ \\
$w_{1}$ & $0.6 \mathrm{~mm}$ & $0.8 \mathrm{~mm}$ & $1.68 \mathrm{~mm}$ \\
$L_{2}$ & $24 \mathrm{~mm}$ & $20 \mathrm{~mm}$ & $40 \mathrm{~mm}$ \\
$\theta_{2}$ & $12^{\circ}$ & $12^{\circ}$ & $11.1^{\circ}$ \\
$w_{2}$ & $4 \mathrm{~mm}$ & $4 \mathrm{~mm}$ & $4 \mathrm{~mm}$ \\
$L_{3}$ & $12 \mathrm{~mm}$ & $8 \mathrm{~mm}$ & $18 \mathrm{~mm}$ \\
$\theta_{3}$ & $0^{\circ}$ & $0^{\circ}$ & $0^{\circ}$ \\
$w_{3}$ & $0.6 \mathrm{~mm}$ & $0.8 \mathrm{~mm}$ & $1.68 \mathrm{~mm}$ \\
\hline
\end{tabular}

the force-deflection characteristics of such an ideal bistable mechanism (the red dash-dot curve) and the corresponding balanced domain (from $y_{1}$ to $y_{2}$ ). As illustrated in Fig. 4, in this ideal balanced domain, the positive stiffness section of the tristable mechanism is compensated by the corresponding part of the negative stiffness section of the bistable mechanism, while its negative stiffness section is compensated by the corresponding positive stiffness section of the bistable mechanism. As the shuttle of the balancing bistable mechanism moves along the $\mathrm{y}$-axis until Part $\mathrm{A}$ of the tristable mechanism switches to its second stable equilibrium position, Part B of the tristable mechanism and the balancing bistable mechanism balance each others stiffness, which results in static balancing of the whole mechanism.

The design of a balancing bistable mechanism hinges upon matching its force-deflection properties with those of the ideal balancing bistable mechanism in the balanced domain, which may be formulated as an optimization problem as follows:

Min IAR $=\int_{y_{1}}^{y_{2}}\left|F_{\mathrm{r}}(y)-F_{\mathrm{i}}(y)\right| d y$

where IAR is the integral of the absolute compensation residual, $F_{\mathrm{r}}(y)$ and $F_{\mathrm{i}}(y)$ are the force-deflection characteristics 


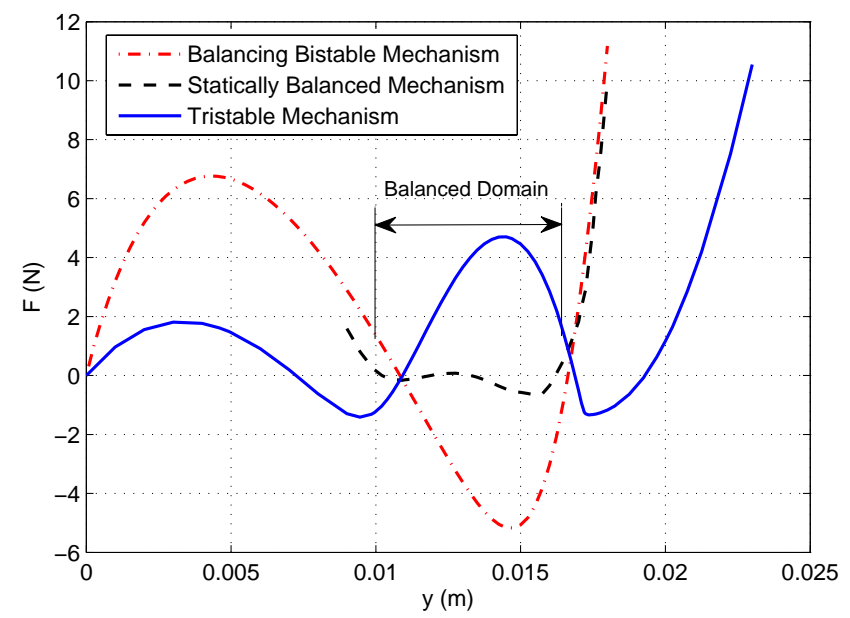

Figure 7. Force-displacement characteristics of the optimized balancing bistable mechanism.

of the candidate and the ideal bistable mechanisms, respectively.

A primary optimization search was conducted using a particle swarm optimizer (PSO) (Chen et al., 2011a) integrated with ANSYS (ANSYS was used to solve the force-deflection characteristics of each candidate bistable mechanism). For simplicity, we assume $L_{1}=L_{3}, w_{1}=w_{3}, w_{2}=4 \mathrm{~mm}, \theta_{1}=\theta_{3}=$ 0 and $H=6 \mathrm{~mm}$ for the balancing bistable mechanism, thus reducing the number of parameters to be optimized to 4 (i.e., $L_{1}, L_{2}, w_{1}$ and $\theta_{2}$ ). In the implementation of PSO, the swarm size is set to 40 and the maximum number of iterations to 200. The optimization process is briefly described as follows:

- Step 1: Randomly initialize 40 particles in the search space, with each particle corresponding to a candidate bistable mechanism.

- Step 2: Evaluate the fitness of each bistable mechanism using Eq. (4).

- Step 3: Update the swarm and generate the next generation particles according to the rules of PSO.

- Step 4: If the current iteration number is less than 200, go to Step 2. Otherwise, the optimization is stopped and the bistable mechanism with the best fitness value is selected as the balancing mechanism for the tristable mechanism.

The optimized parameters of the balancing bistable mechanism are given in Table 1. The resulting force-deflection curves of the tristable and balancing bistable mechanisms (as well as the unbalanced residual force) are plotted in Fig. 7 around the balanced domain.

\subsection{Discussion}

Figure 7 demonstrates that the system is approximately balanced for a specific range of motion around the second stable equilibrium position of the tristable mechanism. Of course, the results can be further improved by conducting a more comprehensive optimization on the balancing bistable mechanism (e.g., taking other design parameters into account). It should be noted that the mechanism must be prestressed into the balanced domain for it to work as a statically balanced device. Statically-balanced mechanisms might be sensitive to fabrication errors (Tolou et al., 2011). We observed the sensitivity of the balancing bistable mechanism to fabrication errors by assuming an error of $5 \%$ is caused during fabrication in both $w_{1}$ and $w_{3}$. Results from our finite element calculations indicate that this causes force-deflection errors of less than $7 \%$ deviated from the ideal. This principle may be easily extended to the static balancing of two multistable mechanisms, each with more than three stable equilibrium positions. In addition, the concept has especial potential for usage in MEMS applications due to its ability to be monolithically fabricated.

\section{Conclusions}

In this paper we have presented two novel concepts which may be used to eliminate the need for prestressing assembly through the use of fully-compliant statically-balanced compliant mechanisms. The first concept utilizes constant-force mechanisms in order to achieve gravity compensation; the second is based upon the combination of two multistable mechanisms in order to achieve static balancing over a certain range of motion (having especial potential for usage in MEMS applications). Each of these concepts has been demonstrated with a case study in which the principles and equations used to formulate them have been shown to perform with good accuracy. Two additional key benefits of these mechanisms is that they can be monolithically fabricated, and they return to their as-fabricated positions without disassembly when they are not in use.

Acknowledgement. The authors gratefully acknowledge the financial support from the National Natural Science Foundation of China under Grant No. 50805110, the Scientific Research Foundation for the Returned Overseas Chinese Scholars under Grant No. JY0600100401, and the Fundamental Research Funds for the Central Universities under No. JY10000904010.

Edited by: J. A. Gallego Sánchez

Reviewed by: two anonymous referees 


\section{References}

Chen, G., Wilcox, D. L., and Howell, L. L.: Fully compliant double tensural tristable micromechanisms (DTTM), J. Micromech. Microeng., 19, 025011, 1-8, 2009a.

Chen, G., Aten, Q. T., Zirbel, S., Jensen, B. D., and Howell, L. L.: A tristable mechanism configuration employing orthogonal compliant mechanisms, Trans. ASME, J. Mechan. Robot., 2, 014501, $1-6,2009 b$.

Chen, G., Gou, Y., and Yang, L.: Research on Multistable Compliant Mechanisms: The State of the Art, Proceedings of the 9th International Conference on Frontiers of Design and Manufacturing (ICFDM 2010), 17-19 July 2010, Changsha, China, 1-5, 2010.

Chen, G., Xiong, B., and Huang, X.: Finding the optimal characteristic parameters for 3R pseudo-rigid-body model using an improved particle swarm optimizer, Precis. Eng., 35(3), 505-511, 2011a.

Chen, G., Gou, Y., and Zhang, A.: Synthesis of compliant multistable mechanisms through use of a single bistable mechanism, Trans. ASME J. Mechan. Design, 133(8), 081007, doi:10.1115/1.4004543, 2011b.

Cherry, B. B., Howell, L. L., and Jensen, B. D.: Evaluating threedimensional effects on the behavior of compliant bistable micromechanisms, J. Micromech. Microeng., 18, 095011, 1-10, 2008.

Gallego, J. A. and Herder, J. L.: Criteria for the static balancing of compliant mechanisms, Proceedings of the ASME Design Engineering Technical Conferences \& Computers and Information in Engineering Conference, Montreal, Canada, 15-18 August 2010, DETC2010-28469, 2010.

Gerson, Y., Krylov, S., Ilic, B., and Schreiber, D.: Large displacement low voltage multistable micro actuator, IEEE 21st International Conference on Micro Electro Mechanical Systems, 463466, 2008

Han, J. S., Muller, C., Wallrabe, U., and Korvink, J. G.: Design, simulation, and fabrication of a quadstable monolithic mechanism with X- and Y-directional bistable curved beams, Trans. ASME J. Mechan. Design, 129, 1198-1203, 2007.

Herder, J. L. and van den Berg, F. P. A.: Statically balanced compliant mechanisms (SBCM's): an example and prospects, Proceedings of the ASME Design Engineering Technical Conferences \& Computers and Information in Engineering Conference, 10 14 September 2000, Baltimore, Maryland, DETC2000/MECH14144, 2000.

Hoetmer, K., Herder, J. L., and Kim, C. J.: A building block approach for the design of statically balanced compliant mechanisms, Proceedings of the ASME Design Engineering Technical Conferences \& Computers and Information in Engineering Conference, San Diego, California, USA, 30 August-18 September 2009, DETC2009-87451, 2009.

Howell, L. L.: Compliant Mechanisms, Wiley-Interscience, New York, NY, 2001.

Jutte, C. V. and Kota, S.: Design of nonlinear springs for prescribed load-displacement functions, Trans. ASME J. Mechan. Design, 130, 081403, 1-10, 2008.

Lan, C.-C., Wang, J.-H., and Chen, Y.-H.: A compliant constantforce mechanism for adaptive robot end-effector operations, Proceedings of the 2010 IEEE International Conference on Robotics and Automation, Anchorage Convention District, 3-8 May, An- chorage, Alaska, USA, 2131-2136, 2010.

Morsch, F. M. and Herder, J. L.: Design of a generic zero stiffness compliant joint, Proceedings of the ASME Design Engineering Technical Conferences \& Computers and Information in Engineering Conference, Montreal, Canada, 15-18 August 2010, DETC2010-28351, 2010.

Nahar, D. R. and Sugar, T.: Compliant constant-force mechanism with a variable output for micro/macro applications, Proceedings of the IEEE International Conference on Robotics and Automation, 1, 318-323, 2003.

Oh, Y. S. and Kota, S.: Synthesis of multistable equilibrium compliant mechanisms using combinations of bistable mechanisms, Trans. ASME J. Mechan. Design, 131, 021002, 1-11, 2009.

Powell, K. M. and Frecker, M. I.: Method for optimization of a nonlinear static balance mechanism, with application to ophthalmic surgical forceps, Proceedings of the ASME 2005 Design Engineering Technical Conferences and Computers and Information in Engineering Conference, 24-28 September 2005, Long Beach, California USA, DETC2005-84759, 2005.

Radaelli, G., Gallego, J. A., and Herder, J. L.: An energy approach to static balancing of systems with torsion stiffness, Proceedings of the ASME Design Engineering Technical Conferences \& Computers and Information in Engineering Conference, Montreal, Canada, 15-18 August 2010, DETC2010-28071, 2010.

Rosenberg, E. J., Radaelli, G., and Herder, J. L.: An energy approach to a 2DOF compliant parallel mechanism with selfguiding statically-balanced straight-line behavior, Proceedings of the ASME Design Engineering Technical Conferences \& Computers and Information in Engineering Conference, Montreal, Canada, 15-18 August 2010, DETC2010-28447, 2010.

Stapel, A. and Herder, J. L.: Feasibility study of a fully compliant statically balanced laparoscopy grasper, Proceedings of the ASME International Design Engineering Technical Conferences and Computers and Information in Engineering Conference, 28 September-2 October 2004, Salt Lake City, Utah, USA, DETC2004-57242, 635-643, 2004.

Tolou, M., Henneken, V. A., and Herder, J. L.: Statically-balanced compliant micro mechanisms (SB-MEMS): concepts and simulation, Proceedings of the ASME Design Engineering Technical Conferences \& Computers and Information in Engineering Conference, Montreal, Canada, 15-18 August 2010, DETC201028406, 2010

Tolou, M., Estevez, P., and Herder, J. L.: Collinear-type statically balanced compliant micro mechanism (SB-CMM): experimental comparison between pre-curved and straight beams, Proceedings of the ASME Design Engineering Technical Conferences \& Computers and Information in Engineering Conference, 2831 August 2011, Washington, DC, USA, DETC2011-47678, in press, 2011.

Trease, B. and Dede, E.: Statically balanced bompliant four-Bar mechanism, ME-599 Design Project Final Report, 2011.

Trease, B. P., Moon, Y.-M., and Kota, S.: Design Of LargeDisplacement Compliant Joints, Trans. ASME J. Mechan. Design, 127, 778-788, 2005.

Wongratanaphisan, T. and Cole, M. O. T.: Analysis of a gravity compensated four-Bar linkage mechanism with linear spring suspension, Trans. ASME J. Mechan. Design, 130, 011006, 1-8, 2008. 